\title{
Mortality and Causes of Death in Patients with Sporadic Inclusion Body Myositis: Survey Study Based on the Clinical Experience of Specialists in Australia, Europe and the USA
}

Mark A. Price ${ }^{\mathrm{a}, *}$, Victoria Barghout ${ }^{\mathrm{b}}$, Olivier Benveniste ${ }^{\mathrm{c}}$, Lisa Christopher-Stine ${ }^{\mathrm{d}}$, Alastair Corbett ${ }^{\mathrm{e}}$, Marianne de Visser $^{f}$, David Hilton-Jones ${ }^{g}$, John T. Kissel ${ }^{\text {h }}$, Thomas E. Lloyd ${ }^{i}$, Ingrid E. Lundberg ${ }^{j}$, Francis Mastaglia $^{\mathrm{k}}$, Tahseen Mozaffar ${ }^{\mathrm{l}}$, Merrilee Needham ${ }^{\mathrm{m}}$, Jens Schmidt ${ }^{\mathrm{n}}$, Kumaraswamy Sivakumar ${ }^{\mathrm{o}}$, Carla DeMuro ${ }^{\mathrm{a}}$ and Brian S. Tseng ${ }^{\mathrm{p}}$

${ }^{a}$ RTI Health Solutions, Research Triangle Park, NC, USA

${ }^{\mathrm{b}}$ VEB HealthCare LLC, Morristown, NJ, USA

${ }^{\mathrm{c}}$ Assistance Publique - Hôpitaux de Paris, Pitié-Salpêtrière University Hospital, Department of Internal Medicine, Pierre and Marie Curie University, Paris, France

${ }^{\mathrm{d}}$ Johns Hopkins Myositis Center, Division of Rheumatology, Johns Hopkins University, Baltimore, MD, USA

e Department of Neurology, Concord Hospital, Concord, NSW, Australia

${ }^{\mathrm{f}}$ Department of Neurology, Academic Medical Center, Amsterdam, Netherlands

${ }^{\mathrm{g}}$ Department of Neurology, John Radcliffe Hospital, Oxford, UK

${ }^{\mathrm{h}}$ Departments of Neurology and Pediatrics, The Ohio State University Wexner Medical Center, Columbus, $O H, U S A$

${ }^{\mathrm{i}}$ Departments of Neurology and Neuroscience, Johns Hopkins University School of Medicine, Baltimore, $M D, U S A$

${ }^{\mathrm{j}}$ Rheumatology Unit, Department of Medicine, Karolinska University Hospital, Solna, Karolinska Institutet, Stockholm, Sweden

${ }^{\mathrm{k}}$ Institute for Immunology and Infectious Diseases, Murdoch University, WA, Australia

${ }^{1}$ Department of Neurology and Orthopedic Surgery, University of California, Irvine, CA, USA

${ }^{\mathrm{m}}$ Western Australian Neuromuscular Research Institute, University of Western Australia, Murdoch University and Department of Neurology, Fiona Stanley Hospital, Perth, WA, Australia

${ }^{\mathrm{n}}$ Department of Neurology, University Medical Center Göttingen; Department of Neuroimmunology, Institute for Multiple Sclerosis Research and Hertie Foundation, University Medical Center Göttingen, Göttingen, Germany ${ }^{\circ}$ Arizona Neurological Research Center, Scottsdale, AZ, USA

${ }^{\mathrm{p}}$ Novartis Pharma Corporation, East Hanover, NJ, USA

\begin{abstract}
.
Background: There is a paucity of data on mortality and causes of death (CoDs) in patients with sporadic inclusion body myositis (sIBM), a rare, progressive, degenerative, inflammatory myopathy that typically affects those aged over 50 years.
\end{abstract}

\footnotetext{
${ }^{*}$ Correspondence to: Mark A. Price, MA, MEd; Senior Director, Surveys and Observational Studies, RTI Health Solutions, 200 Park Offices, PO Box 12194, Research Triangle Park, NC 27709,
}

USA. Tel.: +1 919541 1232; Fax: +1 919485 2704; E-mail: mprice@rti.org. 
Objective: Based on patient records and expertise of clinical specialists, this study used questionnaires to evaluate physicians' views on clinical characteristics of sIBM that may impact on premature mortality and CoDs in these patients.

Methods: Thirteen physicians from seven countries completed two questionnaires online between December 20, 2012 and January 15, 2013. Responses to the first questionnaire were collated and presented in the second questionnaire to seek elaboration and identify consensus.

Results: All 13 physicians completed both questionnaires, providing responses based on 585 living and 149 deceased patients under their care. Patients were reported to have experienced dysphagia (60.2\%) and injurious falls (44.3\%) during their disease. Over half of physicians reported that a subset of their patients with sIBM had a shortened lifespan (8/13), and agreed that bulbar dysfunction/dysphagia/oropharyngeal involvement (12/13), early-onset disease (8/13), severe symptoms (8/13), and falls (7/13) impacted lifespan. Factors related to sIBM were reported as CoDs in $40 \%$ of deceased patients. Oropharyngeal muscle dysfunction was ranked as the leading feature of sIBM that could contribute to death. The risk of premature mortality was higher than the age-matched comparison population.

Conclusions: In the absence of data from traditional sources, this study suggests that features of sIBM may contribute to premature mortality and may be used to inform future studies.

Keywords: Myositis, inclusion body, cause of death, mortality, deglutition disorder, neuromuscular disease, myositis, morbidity

\section{INTRODUCTION}

Sporadic inclusion body myositis (sIBM), also called, inclusion body myositis (IBM), is a progressive, degenerative inflammatory myopathy characterized by slowly progressive weakness and atrophy of muscles of the limbs, face and pharynx [1]. This chronic, debilitating condition affects more men than women (3:1 ratio) [2,3], and in most cases the onset of symptoms occurs after the age of 50 years [3-5]. In the USA and Europe, sIBM is categorized as an orphan condition; nevertheless, sIBM is the most common myopathy in those aged over 50 years [6-8]. The existing literature reports a general population prevalence of 1.1 per million in Turkey [9], 4.9 per million in the Netherlands [10], 9.8 per million in Japan [11], 10.7 per million in the USA [4], 14.9 per million in Western Australia [12], 33 per million in Norway [13] and 50.5 per million in South Australia [14]. It is possible, however, that these figures are underestimates, due to incomplete case ascertainment, misdiagnosis and delayed diagnosis of SIBM [15]. Consistent with this possibility, prevalence estimates have increased over time in follow-up studies $[12,14,16]$, which may reflect improved disease awareness and diagnoses.

Based on the available literature, the most common presenting symptoms of sIBM, reported in over $35 \%$ of patients, are falls and difficulty standing $[1,12$, 15]. In a small proportion of patients, the presentation includes difficulties in performing certain tasks (e.g. gripping, climbing stairs) $[1,15,17]$. As the disease progresses, patients continue to lose muscle strength at a rate of $3.5-16.8 \%$ per year [4, 18-20]; several years after disease onset, most patients require assistance with daily activities, as well as need- ing ambulatory aids such as a cane, walker or wheelchair [1, 4, 19, 21]. Weakening or dysfunction of the pharyngeal and esophageal muscles can lead to dysphagia (difficulty with swallowing), which is experienced by $40-80 \%$ of patients during the course of sIBM, and can result in choking, weight loss, aspiration and pneumonia [1, 5, 19, 20, 22-25]. Currently, there is no approved effective pharmacological treatment for sIBM; physiotherapy, prescribed by most physicians who care for these patients, has been shown to be of some help [26], and some receive local treatment for severe dysphagia [27], as well as general supportive care to manage their symptoms [20].

Few published studies have reported on the natural history of sIBM [4, 18-20, 28-30] or on mortality and causes of death $(\mathrm{CoD})$ associated with the disease $[19,20]$. In both of the studies investigating mortality in patients with sIBM, no significant impact on lifespan compared with the general population was reported $[19,20]$. Furthermore, in a Dutch study comparing patients with sIBM with the age-matched general population, diseases of the respiratory system $(41.3 \%$ of patients with SIBM versus $11.5 \%$ of the general population), and specifically pneumonia (28.3\% versus $4.4 \%$ ), as well as cachexia (severe wasting with loss of weight and muscle mass, $6.5 \%$ versus $0.1 \%$ ), were identified as leading CoDs in individuals in the 80-85 years age category [19]. While these studies provide insights into the clinical course of sIBM, the conclusions that can be drawn are limited by their small sample sizes and short followup times. The paucity of data on the potential impact of sIBM on premature mortality may reflect the challenges of obtaining data on SIBM and other rare diseases from traditional data sources. We therefore 
conducted a survey to evaluate the views of physicians who are treating or have treated patients with sIBM (based on their clinical experience and patient records) in order to assess the contribution of this condition to premature mortality. To the best of our knowledge, this is the largest evaluation of patients with sIBM using this methodology.

\section{MATERIALS AND METHODS}

This study used questionnaires to assess the views of physicians on the risk of premature mortality and CoDs in patients with sIBM, based on their clinical experience and patient records. The study was carried out using a method similar to the Delphi panel technique, which uses iterative rounds of questionnaires to gather relevant data, along with the opinions of experts which are subsequently presented to the respondents to seek consensus [31]. On December 18,2012 , this study was declared exempt from review by the Research Triangle International Committee for the Protection of Human Subjects, owing to the fact that no information that would allow the identification of any patient was ever provided to the study team or the study sponsor by the respondents.

A group of physicians were initially contacted to request their participation in the present survey study. Of those invited, 13 expert physicians (neurologist and/or neuromuscular specialist, $n=10$; rheumatologist, $n=2$; systemic disease specialist, $n=1$ ) from seven countries (Australia, France, Germany, Netherlands, Sweden, UK and USA) who had at least 5 years of experience and were actively treating patients with sIBM responded. The participating physicians were sent a preliminary questionnaire that they did not need to respond to but that invited them to evaluate the medical records of their patients, whom they had diagnosed with sIBM. This was followed by a two-stage questionnaire, for which physicians could use the information that they had gathered during the preliminary questionnaire and did not need to refer to their patient medical records, other than to confirm numbers, where applicable (Supplement, Tables 1-3). The questions were designed to capture summary-level patient data from physicians and their views on premature mortality and CoDs among patients with sIBM, based on their overall clinical experience. The questionnaires were completed online, with physicians able to respond anonymously, at their convenience, within a specified period (December 20-30, 2012 for round 1; January 7-15, 2013 for round 2). The first questionnaire consisted of 20 questions (Supplement, Table 2) and the second questionnaire consisted of 17 questions (Supplement, Table 3). Between rounds 1 and 2 of the process, physician responses were anonymized before being analyzed, collated and summarized; no formal coding or thematic analyses were undertaken. In order to seek consensus, selected collated responses from round 1 , which reflected the views of at least half of the participating physicians, were then presented in round 2. This semi-iterative process was to determine whether the physicians would elaborate on their responses based on information their colleagues had provided. Unique or rare responses from round 1 were not presented in round 2 of the questionnaire.

Responses to the questionnaires were collected as counts or free-text answers. Quantitative data were assessed to calculate means, frequencies and/or standard deviations; for ranking questions, mean scores were calculated and used to rank the relevant features. Qualitative data were assessed by two analysts who independently summarized responses from all participating physicians; the data were compared and any minor discrepancies in qualitative summaries were resolved in order to harmonize the results. The standardized mortality ratio (SMR) with 95\% confidence intervals (CIs) was calculated as the number of deceased patients with sIBM from this dataset divided by the number of deaths that would be expected if the population had the same age-specific mortality as the external comparison population (US general population, based on the 2010 census).

\section{RESULTS}

\section{Patient cohorts}

All 13 physicians completed the questionnaires. Between rounds 1 and 2 of the questionnaire, physicians may have elaborated on their responses, but no physicians changed their response. The responses received were based on 734 patients who had been under the care of the physicians (Table 1). Over onethird of patients were from the USA $(n=298,40.6 \%)$ and over one-quarter were from France $(n=198$, $27.0 \%$ ).

At the time of completing the questionnaire, 585 patients were living and 149 were deceased. Table 2 summarizes the age ranges of living and deceased patients with sIBM who were under the care of the 13 physicians. The majority of living patients were aged $61-80$ years $(61-70$ years, $34.5 \%$; $71-80$ years, $34.9 \%$ ) and the majority of deceased patients 
Table 1

Number of patients under the care of the physicians in each country

\begin{tabular}{lccc}
\hline Country & $\begin{array}{c}\text { Total number of } \\
\text { patients, } n(\%) \\
(N=734)\end{array}$ & $\begin{array}{c}\text { Number of living } \\
\text { patients, } n(\%) \\
(n=585)\end{array}$ & $\begin{array}{c}\text { Number of deceased } \\
\text { patients, } n(\%) \\
(n=149)\end{array}$ \\
\hline Australia (3 physicians) & $94(12.8)$ & $79(13.5)$ & $15(10.1)$ \\
France (1 physician) & $198(27.0)$ & $187(32.0)$ & $11(7.4)$ \\
Germany (1 physician) & $35(4.8)$ & $35(6.0)$ & $0(0.0)$ \\
Netherlands (1 physician) & $19(2.6)$ & $12(2.1)$ & $7(4.7)$ \\
Sweden (1 physician) & $21(2.9)$ & $18(3.1)$ & $3(2.0)$ \\
UK (1 physician) & $69(9.4)$ & $45(7.7)$ & $24(16.1)$ \\
USA (5 physicians) & $298(40.6)$ & $209(35.7)$ & $89(59.7)$ \\
\hline
\end{tabular}

were over 61 years of age $(61-70$ years, $22.8 \%$; $71-80$ years, $36.9 \%$; $>80$ years, $24.8 \%$ ).

\section{Typical clinical characteristics of patients with sIBM}

The typical clinical characteristics of patients with sIBM, based on the experience of the physicians, are summarized in Table 3. The physicians estimated that a lower percentage of their patients had "fastprogressing sIBM" (12.7\%; defined as clinically significant deterioration in limb, bulbar or diaphragmatic strength over 4 months or less) than "slowprogressing sIBM" (77.3\%). The mean reported time from diagnosis to using a cane was 3.9 years; the corresponding time to using a wheelchair was 10.5 years. However, additional information gathered from the free text responses indicated that several physicians found this question difficult to answer owing to the variability in disability levels at the point of sIBM diagnosis, which may be years after the onset of symptoms. Physicians reported that, on average, patients experienced symptoms for 4.5 years before a formal diagnosis was made. Of the patients who had progressed to the point of needing a full-time caregiver, physicians reported that the majority were using an unpaid caregiver $(75.0 \%)$ rather than a professional caregiver or service $(29.0 \%)$.

More than half of the patients were reported to have experienced dysphagia $(60.2 \%)$, a proportion of whom developed aspiration pneumonia (22.7\%). Just fewer than half of the patients were reported to have experienced an injurious fall (44.3\%). Physicians reported that the most common injury experienced by these patients was bruising (stated by $12 / 13$ physicians), followed by broken arm or leg (10/13) and broken hip (9/13).

\section{Features of sIBM that can impact on lifespan}

Physicians were asked to comment on the impact of specific features of sIBM on lifespan. Twelve
Table 2

Age ranges of living and deceased patients under the care of 13 physicians from seven countries

\begin{tabular}{lcc}
\hline Age range & $\begin{array}{c}\text { Number of living } \\
\text { patients } \\
(N=585)\end{array}$ & $\begin{array}{c}\text { Number of deceased } \\
\text { patients } \\
(N=149)\end{array}$ \\
\hline$<40$ years & 2 & 0 \\
41-50 years & 19 & 4 \\
51-60 years & 80 & 19 \\
61-70 years & 202 & 34 \\
$71-80$ years & 204 & 55 \\
$>80$ years & 78 & 37 \\
\hline
\end{tabular}

of the 13 physicians agreed that patients with bulbar dysfunction (abnormal swallowing and speech), dysphagia and oropharyngeal involvement had a shortened lifespan. Over half of the physicians also agreed that patients with early-onset $\operatorname{siBM}(8 / 13)$ and younger patients with more severe symptoms $(8 / 13)$ had a shortened lifespan, particularly among those patients who developed severe dysphagia and weakness leading to immobility. Approximately half of physicians agreed that falls may potentially shorten lifespan (7/13); additional information gathered from free text responses indicated that this was owing to the fact that falls compromised mobility, led to head injuries or had secondary consequences (e.g. sepsis), and were recognized as being more likely to lead to complications in older patients than in younger patients. Ten of the 13 physicians reported that patients with sIBM had a shortened lifespan compared with the general population, a majority of whom (8/10) thought this was only in a subset of patients, primarily those experiencing severe dysphagia.

\section{Clinical characteristics and CoDs among deceased patients with sIBM}

Among deceased patients who were under the care of physicians $(n=149)$, the mean age at diagnosis 
Table 3

Typical clinical characteristics of patients with SIBM based on the experience of 13 physicians from seven countries

\begin{tabular}{|c|c|}
\hline \multicolumn{2}{|l|}{ Characteristics } \\
\hline \multicolumn{2}{|c|}{ Type of sIBM, mean proportion of patients, $\%$ (range) } \\
\hline "Fast progressing"a & $12.7(0-30)^{\mathrm{b}}$ \\
\hline "Slow progressing" & $77.3(20$ \\
\hline \multicolumn{2}{|c|}{ Time from diagnosis to using an ambulatory aid, mean, years } \\
\hline Cane & 3.9 \\
\hline $\mathrm{Wa}$ & \\
\hline lar wheelchair & 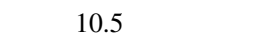 \\
\hline wheelchair & 12.8 \\
\hline \multicolumn{2}{|c|}{ Patients using a caregiver ${ }^{\mathrm{c}}$, mean proportion of patients, $\%$} \\
\hline Professional caregiver/service & 29.0 \\
\hline Unpaid caregiver ${ }^{\mathrm{d}}$ & 75.0 \\
\hline \multicolumn{2}{|c|}{$\begin{array}{l}\text { Clinical characteristics of sIBM that patients have experienced } \\
\text { over the course of illness, mean proportion of patients, \% (range) }\end{array}$} \\
\hline Dysphagia & $60.2(25-90)$ \\
\hline Injurious falls ${ }^{\mathrm{e}}$ & $44.3(10-100)$ \\
\hline \multicolumn{2}{|c|}{$\begin{array}{l}\text { Injuries typically sustained by the patients who have experienced } \\
\text { an injurious fall, number of physicians selecting }\end{array}$} \\
\hline Broken arm or leg & 10 \\
\hline $\mathrm{BrC}$ & 9 \\
\hline Spr & 7 \\
\hline $\mathrm{Br}$ & 2 \\
\hline Head trauma & 8 \\
\hline \multicolumn{2}{|c|}{$\begin{array}{l}\text { aDefined as clinically significant deterioration in limb, bulbar or } \\
\text { diaphragmatic strength over } 4 \text { months or less. "The range reported } \\
\text { in the table for proportion of patients with "fast progressing" } \\
\text { sIBM is based on the following responses: } 0 \% \text { of patients, } 2 \\
\text { physicians; } 5 \% \text { of patients, } 3 \text { physicians; } 10 \% \text { of patients, } 2 \text { physi- } \\
\text { cians; } 20 \% \text { of patients, } 5 \text { physicians; } 30 \% \text { of patients, } 1 \text { physician. } \\
{ }^{c} \text { Expressed as the proportion of patients who have progressed to } \\
\text { the point of needing a full-time caregiver. }{ }^{\mathrm{d}} \text { Unpaid caregiver can } \\
\text { include, but is not limited to, a spouse, child or family member. } \\
\text { e Falls requiring medical attention. sIBM, sporadic inclusion body } \\
\text { myositis. }\end{array}$} \\
\hline
\end{tabular}

was reported to be 62.7 years (range: 60-82 years), and patients had experienced symptoms for a mean of 4.5 years (4-5 years) before diagnosis of sIBM. The mean time between diagnosis and death was 11.2 years (6-20 years).

All CoDs in patients with sIBM (regardless of relationship with sIBM) were reported by physicians based on their clinical experience (Table 4). When physicians were asked to subdivide CoDs according to age group, aspiration and pneumonia were reported to have occurred in all age categories except the 41-50 and 51-60 years age categories (Supplement, Table 4). For approximately $40 \%$ of the deceased patients $(n=57)$, factors related to SIBM were considered to be either a primary CoD (death occurred owing to an event directly related to $\operatorname{sIBM}, n=26$ ) or a secondary $\mathrm{CoD}$ (death did not occur directly owing to sIBM but would probably not have occurred
Table 4

All causes of death in patients with sIBM based on the experience of 13 physicians from seven countries

Cause of death
Respiratory complications (excluding aspiration pneumonia)
Complications arising from dysphagia (including aspiration
pneumonia)
Complications arising from falls
Cardiovascular causes (MI ${ }^{\mathrm{a}}$, chronic heart failure, stroke)
Cancers/malignancy (transitional cell carcinoma, colon cancer,
male breast cancer, leukemia, acute lymphoblastic leukemia,
prostate cancer, lung cancer)
Severe infection due to immunosuppression
Acute sepsis
Leg ulceration
Ulcerative colitis
Renal failure
Cerebrovascular event
Euthanasia
Other systemic diseases
Old age
Unknown

${ }^{\mathrm{a}}$ This abbreviation was not defined by the respondent. ${ }^{\mathrm{b}}$ Reported by a practitioner in the Netherlands for one patient. Euthanasia is legal in the Netherlands, but only for patients experiencing unbearable suffering and when strict procedures are adhered to [19].

if the patient had not have had sIBM, e.g. dysphagia giving rise to aspiration and pneumonia, $n=31$ ). However, in approximately $40 \%$ of deceased patients $(n=54)$, the CoD was not thought to be associated with factors related to sIBM. Physicians ranked features of sIBM that could have contributed to the death of the deceased patients who were under their care (Fig. 1) and reported the most common factor to be oropharyngeal muscle dysfunction. The most commonly reported end-of-life setting for deceased patients with SIBM was the home or a nursing home, whereas the least common setting was a rehabilitation center (Fig. 2).

\section{Risk of premature mortality in patients with sIBM}

Based on this dataset, patients with sIBM who were aged 41 years and older had an approximately 7-fold higher risk of premature mortality than the age-matched external comparison population (SMR, 6.58; 95\% CI, 5.6-7.7). As expected, the risk of premature mortality in patients with sIBM decreased with increasing age, but was still approximately 5-fold higher than the age-matched external comparison population in patients over 70 years of age (SMR, 4.82; 95\% CI, 3.9-5.9). 


\begin{tabular}{|c|c|c|c|c|c|}
\hline $\begin{array}{c}\text { Oropharyngeal } \\
\text { muscle } \\
\text { dysfunction }\end{array}$ & $\begin{array}{c}\text { General } \\
\text { debility }\end{array}$ & $\begin{array}{c}\text { Respiratory } \\
\text { muscle } \\
\text { dysfunction }\end{array}$ & $\begin{array}{c}\text { Limb/axial } \\
\text { muscle } \\
\text { dysfunction }\end{array}$ & Comorbidities & $\begin{array}{c}\text { Psychiatric } \\
\text { (suicide) }\end{array}$ \\
$\begin{array}{c}1 \\
\text { Most }\end{array}$ & 2 & 3 & 4 & 5 & 6 \\
common & & & 5 & Least \\
common
\end{tabular}

Fig. 1. Ranked features of sIBM that can contribute to death, based on the experience of 13 physicians from seven countries. Features presented for ranking were based on the physicians' responses in round 1 of the questionnaire. Each physician was asked to score the features of sIBM shown above on the basis of contribution to death in the patients they were following $(1=$ most common, $5=$ least common). Five physicians did not think features of sIBM contributed to death. Of those physicians who provided scores, mean scores were calculated and used to rank features. Physicians did not expand on which comorbidities associated with sIBM could contribute to death. sIBM, sporadic inclusion body myositis.

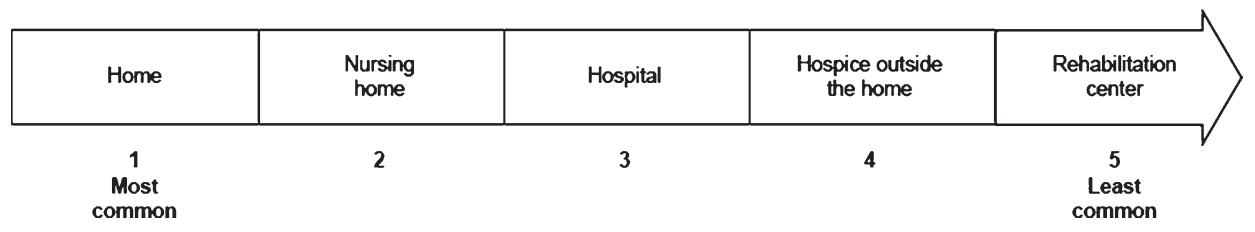

Fig. 2. Ranked end-of-life settings for patients with sIBM, based on the experience of 13 physicians from seven countries. Each physician was asked to score the end-of-life settings shown above ( $1=$ most common, $5=$ least common). Based on all the physicians' responses, the mean score was calculated and used to rank the settings. sIBM, sporadic inclusion body myositis.

\section{DISCUSSION}

This study, which used questionnaires, was designed to capture the clinical experience of 13 physicians based on their patient records and direct clinical contact with patients with $\operatorname{SIBM}(734$ patients with sIBM in total). The questionnaires assessed clinical characteristics of sIBM that could contribute to premature mortality and CoDs in patients with SIBM, based on the responses of physicians in Australia, Europe and the USA. This analysis revealed that physicians are of the view that a large proportion of their patients experience comorbidities that could be attributed to SIBM and that features of SIBM may impact on the risk of premature mortality in $40 \%$ of their patients $(n=57)$.

The physicians in this study were asked to describe the typical clinical characteristics of their patients. Consistent with the description of SIBM as a slowly progressive weakness and atrophy of muscles, the majority of patients were categorized as having "slow-progressing sIBM" [1]. In this study, the time to using a cane or wheelchair was reported to be 3.9 and 10.5 years, respectively, which is shorter than that reported in other studies (walking stick, 11 years; wheelchair, 13-16 years) $[5,19,20]$. This difference may be explained by the fact that the present study assessed time since diagnosis of sIBM, while other studies assessed time since symptom onset; it is well known that there can be a long period between the onset of symptoms and the diagnosis of sIBM (4.5 years in this study) [20, 22]. If we account for the average time that patients experienced symptoms before formal diagnosis, the time to using an ambulatory aid is similar between the present study and previously reported observations. The physicians reported that $60 \%$ of their patients had experienced dysphagia in the course of their illness, which is within the range reported by several other studies (20-80\%) [1, 5, 10, 19, 22-24, 32]. Nearly half of the patients (44\%) were also reported to have experienced injurious falls, which is lower than the proportion of patients reporting frequent falls in a study by Badrising et al. (73\%) [5]. However, this may reflect differences in the patient populations or in the definition used to assess falls (the proportion of patients experiencing injurious falls versus the proportion of patients experiencing periods of frequent falls). Overall, the prevalence of clinical characteristics of sIBM reported for the population described in this study (e.g. dysphagia, falls) were generally within the range reported in other studies, supporting the present questionnaire-based methodology to assess CoDs and risk of premature mortality in patients with SIBM, for which there is a paucity of data.

The majority of the physicians in the present study reported that a subset of patients with sIBM had a shortened lifespan. Nearly all the physicians agreed that patients with bulbar dysfunction, dysphagia or 
oropharyngeal involvement, which are features of sIBM, impacted on lifespan. Furthermore, over half of the physicians agreed that younger patients experiencing severe symptoms and those with early disease onset had a shortened lifespan, particularly among patients who developed dysphagia or who had weakness leading to immobility. Over half of physicians also agreed that patients experiencing falls had a shortened lifespan, particularly older patients and cases where falls led to head injuries, secondary complications (e.g. sepsis) or immobility. Given that $40-60 \%$ of patients experience dysphagia (a proportion of whom experience severe dysphagia) or injurious falls, the present study suggests that lifespan may be affected in a large proportion of patients. In a recent study of Dutch patients with sIBM who died, lifespan was not reported to be shortened versus the Dutch general populations [19]. A similar finding was reported in a study of French and British patients (using the French general population as the reference population) [20]. The discrepancy between the current study and the Dutch and French/British studies may be due to differences in study design, patient populations, or the small sample sizes being investigated ( $n=25-46)$. Furthermore, Cox et al. and Benveniste et al. assessed lifespan in the entire SIBM population in their studies, and did not consider subsets of patients (e.g. those with severe symptoms) in whom lifespan may have been affected by sIBM.

While sIBM is rarely considered a direct $\mathrm{CoD}$, it is recognized that some deaths may be related to complications of the disease (e.g. pneumonia arising from dysphagia) $[19,20]$. Indeed, in the present study, physicians considered factors related to SIBM to have contributed to the death of almost half of the deceased patients under their care, and the risk of premature mortality was 5-7-fold higher in patients with sIBM than in the age-matched comparison population. The highest-ranked feature of sIBM considered to contribute to death was oropharyngeal muscle dysfunction, which can lead to dysphagia and subsequently aspiration and pneumonia [19, 20, 22]. Consistent with this, aspiration and pneumonia were reported as CoDs in the 61-70 years, 71-80 years and $>80$ years age categories, which contained almost $85 \%$ of the patients under the care of the physicians. Results on CoD in the present study are consistent with those of Cox et al., who reported that diseases of the respiratory system, including pneumonia and cachexia, all of which can arise from dysphagia, were leading CoDs in the sIBM cohort versus the general population in the $80-85$ years age category [19]. While these studies highlight leading CoDs in patients with sIBM, further studies are needed to more fully investigate CoDs in these patients, including estimates of their relative frequency.

To date, there is a paucity of information on mortality and CoD in patients with sIBM. This probably reflects the difficulty of capturing these data in patients with this rare disease. Until 2010, sIBM did not have an International Classification of Diseases, Ninth Revision, Clinical Modification (ICD-9-CM) code; therefore, traditional data sources (e.g. medical records) would have reported only conditions related to sIBM, rather than SIBM itself (as a diagnosis or $\mathrm{CoD}$ ). Furthermore, owing to the protracted nature of sIBM, patients are likely to be treated by several different physicians, so retrospective studies of medical records would not necessarily provide a patient's complete medical history. To overcome these issues, this study used questionnaires to assess the views of physicians based on their broad clinical experience and is, to the best of our knowledge, the largest assessment of patients with sIBM using this approach. The methodology ensured that all physicians had an equal voice to express their views and, using two iterations of enquiry, provided the opportunity to seek elaboration or identify consensus. The findings of this study provide important insights into the real-world views of physicians with different specialties in different countries on the burden of sIBM, and can be used to support and contextualize studies performed using traditional data sources. Future studies are needed to comprehensively assess potential risks of sIBM and further investigate complications that could arise from features of the disease, such as sleep disordered breathing as a result of oropharyngeal muscle dysfunction $[33,34]$ or injuries arising from falls.

There are a number of limitations of this study that may have introduced bias, such as the fact that the findings were based on information from 13 physicians from only seven countries, with variable clinical experience of sIBM. While the physicians were requested to assess patient clinical records before completing the questionnaires, physicians were not required to cross-check their responses against their clinical charts. The information reported may therefore have been influenced by impressions from their experience and recall bias, with a potential to have overestimated the frequency of severely affected patients. For those physicians following only a few patients with sIBM, or with limited number of years in practice, experience may not completely reflect 
the actual real-world situation. Furthermore, owing to the nature of data collection, some information may not have been captured. For example, severely affected patients with sIBM are more likely than those with mild or moderately affected disease to be lost at follow-up at tertiary centers owing to the difficulty of transporting them. However, to overcome these limitations, the methodology assessed the average views of the physicians and sought consensus among them, where appropriate, in part to gain a better understanding of the consistency of views between the physicians. Another limitation is that patients within a country may have moved between the recruited physicians over the course of their disease, and there is therefore a small but existing possibility that data for some patients may have been duplicated. However, this does not reduce the significance or impact of the findings, which were based on summary-level patient data and the general views of the physicians. Finally, the SMR data should be interpreted with caution owing to the large variation in patient mortality recorded by the participating physicians.

\section{CONCLUSION}

This study provides important insights into the clinical characteristics and CoDs in patients with sIBM. It suggests an increased risk of mortality in these patients relative to an age-matched comparison population, particularly in those with impaired swallowing and subsequent aspiration and pneumonia. The findings complement and contextualize what is already known in the field, as well as providing novel insights into the features of sIBM that physicians view as contributing to premature mortality. The lack of unanimity among physicians in their responses to some questions highlights the need for further prospective studies, preferably those that are population-based and have longitudinal follow-up, to confirm the findings of the present study. Nevertheless, these results highlight that there is a recognized need to manage the consequences of sIBM, primarily severe dysphagia, in order to reduce their potential contribution to premature mortality.

\section{ACKNOWLEDGMENTS}

The authors take full responsibility for the content of the paper. The authors thank Dr Anne-Marie Couto and Dr Gemma Carter (Oxford PharmaGenesis. Oxford, UK) for medical writing support, editorial assistance, and collation and incorporation of comments from all authors (funded by Novartis Pharma AG, Basel, Switzerland). The authors would also like to acknowledge Gorana Capkun (Novartis Pharma AG, Basel, Switzerland) for provision of advice and guidance when developing the manuscript, and Paola Primatesta (Novartis Pharma AG, Basel, Switzerland) and Estel Plana (formerly Novartis Pharma AG, Barcelona, Spain) who performed the SMR analysis.

\section{FUNDING}

This study was funded by Novartis Pharma AG, Basel, Switzerland.

\section{CONFLICTS OF INTEREST}

MA Price and C DeMuro are employees of RTI Health Solutions. V Barghout is an employee of VEB HealthCare LLC. RTI Health Solutions and VEB HealthCare were paid by Novartis Pharma AG. $O$ Benveniste received honoraria, research grants or reimbursements for travel from Genzyme, Shire, LFB Biopharmaceuticals, CSL Behring, Neovacs and Novartis. L Christopher-Stine is a site co-investigator for a Novartis-sponsored clinical trial, has received consulting fees from Novartis and has served as an advisory board member and/or has received consulting fees from Walgreen's, Mallinckrodt, Idera and Medimmune/Astra Zeneca. M de Visser is a site co-investigator for a Novartis-sponsored clinical trial, has served as an advisory board member and/or has received consulting fees from Medimmune and Lilly. TE Lloyd is a site principal investigator for a Novartis-sponsored clinical trial and has received consulting fees from Novartis. IE Lundberg has received a research grant from Bristol-Myers Squibb and Astra Zeneca, has served on advisory boards of Novartis, aTyr Inc, Idera Inc and has stock shares in Pfizer Inc. M Needham has received honoraria or reimbursements for travel from Bayer, Biogen Idec and Novartis. J Schmidt received honoraria, research grants or reimbursements for travel from Bayer, Biogen, BioMarin, Biotest, CSL Behring, Grifols, Novartis, Octapharma and VitalAire. BS Tseng is a paid employee of Novartis Pharma Corporation. A Corbett, D Hilton-Jones, JT Kissel, F Mastaglia, T Mozaffar and K Sivakumar have no conflict of interest to report. 


\section{SUPPLEMENTARY MATERIAL}

The supplementary material is available in the electronic version of this article: http://dx.doi.org/ 10.3233/JND-150138.

\section{REFERENCES}

[1] Dalakas MC. Inflammatory muscle diseases. N Engl J Med. 2015;372:1734-47.

[2] Dimachkie MM, Barohn RJ. Inclusion body myositis. Semin Neurol. 2012;32(3):237-45.

[3] Lotz BP, Engel AG, Nishino H, Stevens JC, Litchy WJ. Inclusion body myositis. Observations in 40 patients. Brain. 1989;112(Pt 3):727-47.

[4] Felice KJ, North WA. Inclusion body myositis in Connecticut: Observations in 35 patients during an 8-year period. Medicine (Baltimore). 2001;80(5):320-7.

[5] Badrising UA, Maat-Schieman ML, van Houwelingen JC, van Doorn PA, van Duinen SG, van Engelen BG, et al. Inclusion body myositis. Clinical features and clinical course of the disease in 64 patients. J Neurol. 2005;252(12):1448-54.

[6] Salajegheh M, Rakocevic G, Raju R, Shatunov A, Goldfarb LG, Dalakas MC. T cell receptor profiling in muscle and blood lymphocytes in sporadic inclusion body myositis. Neurology. 2007;69(17):1672-9.

[7] Salajegheh M, Raju R, Schmidt J, Dalakas MC. Upregulation of thrombospondin-1(TSP-1) and its binding partners, CD36 and CD47, in sporadic inclusion body myositis. J Neuroimmunol. 2007;187(1-2):166-74.

[8] Lunemann JD, Schmidt J, Schmid D, Barthel K, Wrede A, Dalakas MC, et al. Beta-amyloid is a substrate of autophagy in sporadic inclusion body myositis. Ann Neurol. 2007;61(5):476-83.

[9] Oflazer PS, Deymeer F, Parman Y. Sporadic-inclusion body myositis (s-IBM) is not so prevalent in Istanbul/Turkey: A muscle biopsy based survey. Acta Myol. 2011;30(1):34-6.

[10] Badrising UA, Maat-Schieman M, van Duinen SG, Breedveld F, van Doorn P, van Engelen B, et al. Epidemiology of inclusion body myositis in the Netherlands: A nationwide study. Neurology. 2000;55(9):1385-7.

[11] SuzukiN, Aoki M, Mori-Yoshimura M, Hayashi YK, Nonaka I, Nishino I. Increase in number of sporadic inclusion body myositis (sIBM) in Japan. J Neurol. 2012;259(3):554-6.

[12] Needham M, Corbett A, Day T, Christiansen F, Fabian V, Mastaglia FL. Prevalence of sporadic inclusion body myositis and factors contributing to delayed diagnosis. J Clin Neurosci. 2008;15(12):1350-3.

[13] Dobloug GC, Antal EA, Sveberg L, Garen T, Bitter H, Stjarne J, et al. High prevalence of inclusion body myositis in Norway; a population-based clinical epidemiology study. Eur J Neurol. 2015;22(4):672-e41.

[14] Tutuncu M, Tang J, Zeid NA, Kale N, Crusan DJ, Atkinson EJ, et al. Onset of progressive phase is an agedependent clinical milestone in multiple sclerosis. Mult Scler. 2013;19(2):188-98.

[15] Mastaglia FL. Sporadic inclusion body myositis: Variability in prevalence and phenotype and influence of the MHC. Acta Myol. 2009;28(2):66-71.

[16] Phillips BA, Zilko PJ, Mastaglia FL. Prevalence of sporadic inclusion body myositis in Western Australia. Muscle Nerve. 2000;23(6):970-2.

[17] Needham M, James I, Corbett A, Day T, Christiansen F, Phillips B, et al. Sporadic inclusion body myositis:
Phenotypic variability and influence of HLA-DR3 in a cohort of 57 Australian cases. J Neurol Neurosurg Psychiatry. 2008;79(9):1056-60.

[18] Lindberg C, Persson LI, Bjorkander J, Oldfors A. Inclusion body myositis: Clinical, morphological, physiological and laboratory findings in 18 cases. Acta Neurol Scand. 1994; 89(2):123-31.

[19] Cox FM, Titulaer MJ, Sont JK, Wintzen AR, Verschuuren JJ, Badrising UA. A 12-year follow-up in sporadic inclusion body myositis: An end stage with major disabilities. Brain. 2011;134(Pt 11):3167-75.

[20] Benveniste O, Guiguet M, Freebody J, Dubourg O, Squier W, Maisonobe T, et al. Long-term observational study of sporadic inclusion body myositis. Brain. 2011;134(Pt 11):3176-84.

[21] Machado P, Miller A, Holton J, Hanna M. Sporadic inclusion body myositis: An unsolved mystery. Acta Reumatol Port. 2009;34(2A):161-82.

[22] Amato AA, Barohn RJ. Inclusion body myositis: Old and new concepts. J Neurol Neurosurg Psychiatry. 2009;80(11): 1186-93.

[23] Houser SM, Calabrese LH, Strome M. Dysphagia in patients with inclusion body myositis. Laryngoscope. 1998;108(7): 1001-5.

[24] Oh TH, Brumfield KA, Hoskin TL, Kasperbauer JL, Basford JR. Dysphagia in inclusion body myositis: Clinical features, management, and clinical outcome. Am J Phys Med Rehabil. 2008;87(11):883-9.

[25] Lundberg IE, Forbess CJ. Mortality in idiopathic inflammatory myopathies. Clin Exp Rheumatol. 2008;26(5 Suppl 51):S109-14.

[26] Munters LA. New insights into the benefits of exercise for muscle health in patients with idiopathic inflammatory myositis. Curr Rheumatol Rep. 2014;16(7):429.

[27] Carstens PO, Schmidt J. Diagnosis, pathogenesis and treatment of myositis: Recent advances. Clin Exp Immunol. 2014;175(3):349-58.

[28] Hogrel JY, Allenbach Y, Canal A, Leroux G, Ollivier G, Mariampillai K, et al. Four-year longitudinal study of clinical and functional endpoints in sporadic inclusion body myositis: Implications for therapeutic trials. Neuromuscul Disord. 2014;24(7):604-10.

[29] Rose MR, McDermott MP, Thornton CA, Palenski C, Martens WB, Griggs RC. A prospective natural history study of inclusion body myositis: Implications for clinical trials. Neurology. 2001;57(3):548-50.

[30] Dalakas MC, Rakocevic G, Schmidt J, Salajegheh M, McElroy B, Harris-Love MO, et al. Effect of Alemtuzumab (CAMPATH 1-H) in patients with inclusion-body myositis. Brain. 2009;132(Pt 6):1536-44.

[31] Dalkey N, Helmer O. An experimental application of the Delphi method to the use of experts. Health Technol Assess. 1963;2:458-67.

[32] Munshi SK, Thanvi B, Jonnalagadda SJ, Da Forno P, Patel A, Sharma S. Inclusion body myositis: An underdiagnosed myopathy of older people. Age Ageing. 2006;35(1):91-4.

[33] Rodriguez Cruz PM, Needham M, Hollingsworth P, Mastaglia FL, Hillman DR. Sleep disordered breathing and subclinical impairment of respiratory function are common in sporadic inclusion body myositis. Neuromuscul Disord. 2014;24(12):1036-41.

[34] Della Marca G, Sancricca C, Losurdo A, Di Blasi C, De Fino C, Morosetti R, et al. Sleep disordered breathing in a cohort of patients with sporadic inclusion body myositis. Clin Neurophysiol. 2013;124(8):1615-21. 